Research Paper

\title{
Association of physical performance and biochemical profile of mice with intrinsic endurance swimming
}

\author{
Wen-Ching Huang1, Yi-Ju Hsu ${ }^{1}$, Li Wei ${ }^{2}$, Ying-Ju Chen ${ }^{3 凶}$, Chi-Chang Huang ${ }^{1 \bowtie}$ \\ 1. Graduate Institute of Sports Science, National Taiwan Sport University, Taoyuan 33301, Taiwan. \\ 2. Department of Neurosurgery, Taipei Medical University-Wan Fang Hospital, Taipei 11696, Taiwan. \\ 3. Department of Food and Nutrition, Providence University, Taichung City 43301, Taiwan. \\ $\triangle$ Corresponding authors: Graduate Institute of Sports Science, National Taiwan Sport University, No. 250, Wenhua 1st Rd., Guishan Township, Taoyuan \\ County 33301, Taiwan (C.-C.H.); Department of Food and Nutrition, Providence University, 200 Chung-Chi Rd., Salu Dist., Taichung City 43301, Taiwan \\ (Y.-J.C.). Tel.: +886-3-328-3201 (ext. 2619) (C.-C.H.); +886-4-26328001 (ext. 15031) (Y.-J.C.). Email: john5523@ntsu.edu.tw (C.-C.H.); yjchen5@pu.edu.tw (Y.-J.C.).
}

() Ivyspring International Publisher. Reproduction is permitted for personal, noncommercial use, provided that the article is in whole, unmodified, and properly cited. See http://ivyspring.com/terms for terms and conditions.

Received: 2016.06.08; Accepted: 2016.08.22; Published: 2016.11.02

\begin{abstract}
We aimed to investigate the potential mediators and relationship affecting congenital exercise performance in an animal model with physical activity challenge from physiological and biochemical perspectives. A total of 75 male ICR mice (5 weeks old) were adapted for 1 week, then mice performed a non-loading and exhaustive swimming test and were assigned to 3 groups by exhaustive swimming time: low exercise capacity (LEC) $(<3 \mathrm{hr})$, medium exercise capacity (MEC) (3-5 hr), and high exercise capacity (HEC) ( $>5 \mathrm{hr}$ ). After a 1-week rest, the 3 groups of mice performed an exhaustive swimming test with a $5 \%$ and $7.5 \%$ weight load and a forelimb grip-strength test, with a 1 -week rest between tests. Blood samples were collected immediately after an acute exercise challenge and at the end of the experiment (resting status) to evaluate biochemical blood variables and their relation with physical performance. Physical activity, including exhaustive swimming and grip strength, was greater for HEC than other mice. The swimming performance and grip strength between groups were moderately correlated $(r=0.443$, $p<0.05)$. Resting serum ammonium level was moderately correlated with endurance with a $7.5 \%$ weight load $(r=-0.447, p<0.05)$ and with lactate level $(r=0.598, p<0.05)$. The pulmonary morphology of the HEC group seemed to indicate benefits for aerobic exercise. Mice showed congenital exercise performance, which was significantly correlated with different physical challenges and biochemical variable values. This study may have implications for interference in intrinsic characteristics
\end{abstract}

Key words: Congenital, Exercise performance, Endurance swimming, Forelimb grip, Ammonia.

\section{Introduction}

A heterogeneous stock was produced from the same 8 founder strains, the Collaborative Cross (CC) inbred strains, which include A/J, C57BL/6J, 129S1/SvImJ, NOD/ShiLtJ, NZO/H1LtJ, CAST/EiJ, $\mathrm{PWK} / \mathrm{PhJ}$, and WSB/EiJ [1]. The genetically heterogeneous diversity outbred mice showed a broad range of phenotypes, heterozygosity, and genetic buffering. The advantages of outbreeding are normal levels of heterozygosity, similarity to the genetic condition in humans, and substantially increased mapping resolution [2]. The outbred mice showed good adaption, healthy resistance, reproductive ability, and easy management. They are usually used in studies of toxicological assessment, embryology and physiology [3-5].

For sport science with animal experiments, exercise performance is usually evaluated by two exercise models: treadmill running and exhaustive swimming test. The treadmill test forces experimental animals to run according to an increased gradient of speed or slope by a negative motivation, electric shock, until exhaustion [6]. The exhaustive swimming 
test involves a loading equivalent to body weight on the tail and is based on the animal's survival instinct [7]. The period of physical activity from the beginning to exhaustion is considered exercise performance.

In recent years, many studies have focused on the effects of different nutrient supplements on physical fatigue or related physiology. Outbred rodents such as Sprague-Dawley rats and ICR and ddY mice are widely used in sport science [8-12]. Biochemical variables including lactate, ammonia, glucose, and creatine kinase have been evaluated for fatigue status. During or after intensive exercise, lactate and ammonia levels are accumulated to cause deleterious effects and result in fatigue [13]. Simultaneously, the muscle needs a great quantity of energy, and lactate level is increased with anaerobic glycolysis. The accumulated lactate level further reduces $\mathrm{pH}$ value, which could affect the enzyme activity of glycolysis and muscle contraction via calcium ion release [14]. The immediate ammonia level produced from deamination of AMP to AIP via the purine nucleotide cycle is greatest during intensive exercise, when the rate of ATP utilization may exceed that of ATP production. It could cause peripheral and central fatigue because of muscle phosphocreatine depletion, proton accumulation in muscle, muscle glycogen depletion, reduced blood glucose concentration, and increased ratio of specific amino acids in plasma [15-16].

The intrinsic capacity of rodents can be artificially bred to produce stable inherited lines for basic studies with different purposes. Using a treadmill running protocol, Koch et al. found that the founder population could be divided by high and low exercise capacity, with endurance capacity greater for the high-capacity than low-capacity line by 1.7 times after 6 generations of breeding [17]. As well, artificial selection for high voluntary activities could show significant behavioral differences due to differences in neurotransmitter-associated metabolites in brain regions that affect exercise and motivational state [18]. In the low-capacity line, a combination of risk factors, including female sex and overweight, promoted myocardial stiffness and fibrosis sufficient to cause diastolic dysfunction [19]. In another report, left ventricular myocardial and cardiomyocyte morphology, contractility, and intracellular $\mathrm{Ca}^{2+}$ handling in both systole and diastole, as well as mean blood pressure, were compromised in rats bred for low aerobic capacity during progression from adult to old age [20]. The high-capacity line did not differ from the low-capacity line in arteriolar branching geometry of the heart and skeletal muscle. Therefore, endothelial tolerance for shear stress and/or coupling of myocardial muscle fiber contractile function were suggested to be increased because of allowing higher flow rate per unit volume muscle or more efficient use of oxygen and nutrients [21].

In established high- or low-capacity animal lines, previous reports addressed the physiological or pathological differences but not biochemical variables and correlations by intensity of exercise. In our previous studies, we found that the same batch of outbred mice exhibited a great variety in exercise performance, so we evaluated the individual mouse exercise capacity before supplement intervention. We have limited reports to reveal the causes or physiological correlations of congenital exercise performance. Therefore, the primary aim of our investigation was to test whether exhaustive swimming and treadmill running tests could be used to distinguish intrinsic exercise capacity in mice. The secondary aim was to determine whether biochemical characteristics were associated with different physical activity intensity in mice with different capacities. This study could provide insights for researchers in sport science who use an animal model to study interference in intrinsic exercise differences. Nutrient supplements or interventions could substantially affect exercise performance or physiology.

\section{Methods}

\section{Animal and experimental designs}

Male outbred ICR mice (5 weeks old) were purchased from BioLASCO (A Charles River Licensee Corp., Yi-Lan, Taiwan). All animals were maintained under $23 \pm 2{ }^{\circ} \mathrm{C}$ room temperature, $55 \pm 10 \%$ humidity, and provided a standard laboratory diet (\#. 5001; PMI Nutrition International, Brentwood, MO, USA), with distilled water ad libitum. Mice were adapted to the environment for at least 1 week before experiments. The experimental animals were raised in the animal room of National Taiwan Sport University (NTSU) and all procedures were approved by the Institutional Animal Care and Use Committee of NTSU (IACUC-10103).

After 1-week adaption, all 75 mice performed the exhaustive swimming exercise with $0 \%, 5 \%, 7.5 \%$ body-weight loading on the tail and a grip strength test, with a 1-week rest interval between each physical activity. After the $0 \%$ loading weight load evaluation, mice were assigned to 3 groups by exhaustive swimming time: low exercise capacity (LEC) $(<3 \mathrm{hr})$, medium exercise capacity (MEC) (3-5 hr), and high exercise capacity (HEC) $(>5 \mathrm{hr})$. Each group comprised an equal proportion of about 24-27 mice for the following tests. One week after the last grip strength test, 8 mice randomly chosen from each group were killed. Blood was collected, and organs 
including the heart, lung, liver, muscle, epididymal adipose tissue (EAT), and brown adipose tissue (BAT) were collected and weighed for further biochemical and pathological analysis.

\section{Exhaustive swimming exercise}

A weight equivalent to $0 \%, 5 \%$, and $7.5 \%$ body weight was attached to the root of the mouse tail by lead fish sinkers. The tank was maintained at $28^{\circ} \mathrm{C}$ during the swimming process and the endurance for each mouse was measured as swimming time recorded from the beginning of the time to exhaustion, defined by observing uncoordinated movements and failure to return to the surface within 7 s. The time of floating, struggling and making necessary movements was assessed until possible drowning and exhaustion. The other details of the exhaustive swimming exercise were previously described $[7,9]$.

\section{Forelimb grip strength}

The low-force testing system (Model-RX-5, Aikoh Engineering, Nagoya, Japan) was used to measure forelimb grip strength. The amount of tensile force was measured by the force transducer equipped with a metal bar (2 $\mathrm{mm}$ diameter, $7.5 \mathrm{~cm}$ length), which was gripped by mice. The maximal force (grams) recorded by the low-force system was considered grip strength. The detailed procedures were previously described [7].

\section{Blood biochemical variables}

The biochemical variables lactate, ammonia, and glucose were evaluated for fatigue status in previous studies [7, 9]. We evaluated these variables for possible association with congenital exercise performance. Blood samples were immediately collected from resting mice by means of a submandibular duct method in fed mice. For the effects of physical challenge on these variables, blood samples were immediately collected from the mice after the 15-min swimming test without weight loading. The plasma was analyzed for lactate, ammonia, glucose, and creatine kinase levels by use of an auto-analyzer (Hitachi 7060, Hitachi, Tokyo) after centrifugation at $1,500 \times \mathrm{g}, 4^{\circ} \mathrm{C}$ for $10 \mathrm{~min}$.

\section{Oral glucose tolerance test (OGTT)}

The mice fasted for $16 \mathrm{hr}$ before oral glucose challenge. The glucose $(2.5 \mathrm{~g} / \mathrm{kg})$ was administered by oral gavage and blood samples were collected from the tail vein at $0,15,30,60$, and $120 \mathrm{~min}$ for glucometer analysis (ACCU-CHEK Comfort, Roche).

\section{Histology}

The mice were euthanized with $\mathrm{CO} 2$ in an appropriate chamber by trained personnel at the end of experiments. The blood was collected, and tissues or organs related to physical activities or energy homeostasis were weighed and immersed in 10\% buffered formalin. Paraffin-embedded samples were sectioned into 4- $\mu \mathrm{m}$-thick slides and underwent hematoxylin and eosin (H\&E) staining as previously described [22]. Samples were photographed by using a light microscope equipped with a CCD camera (BX-51, Olympus, Tokyo).

\section{Statistical analysis}

All data are expressed mean \pm SEM and were analyzed by use of SAS 9.0 (SAS Inst., Cary, NC, USA). The Cochran-Armitage test for dose-effect trend analysis was evaluated by use of SAS v9.0. The performance in the exhaustive swimming exercise with $0 \%, 5 \%$, and $7.5 \%$ loading equivalent to body weight, grip strength and biochemical variables were assessed by one-way ANOVA. The correlation between exhaustive swimming exercise $(0 \%, 5 \%$, and $7.5 \%$ loading), grip strength, and biochemical variables was analyzed by Pearson's product-moment correlation coefficient (r). $P<0.05$ was considered statistically significant.

\section{Results}

\section{Exercise performance, definition, general characteristics}

After 1-week adaption, all mice performed the exhaustive swimming exercise with $0 \%$ body-weight loading. The endurance of individual mice exhibited normal contribution and significant difference according to number of mice and endurance time (Fig. 1a). We ranked mice by individual swimming time (Fig. 1b). Most mice were exhausted within $10 \mathrm{hr}$, and the mean exhaustive swimming time was about $4 \mathrm{hr}$. However, a few mice exhibited extreme endurance capacity, more than $10 \mathrm{hr}$. Therefore, we divided all mice into 3 experimental groups (LEC, MEC, and HEC) according to swimming endurance time: $<3 \mathrm{hr}$, 3-5 hr, and $>5 \mathrm{hr}$, respectively. Each group contained 24-27 mice for further physical tests (Table 1). To monitor the growth curve and nutritional supplement, we recorded the body weight and daily intake of each group. The 3 groups did not differ in weight, diet or water intake at each week (Table 2). Therefore, the difference in exercise performance would not be affected by nutritional supplementation and might be affect by other physiological factors.

\section{Physical activity test}

The physical activities in this experiment included an exhaustive swimming exercise with $0 \%$, $5 \%$, and $7.5 \%$ body weight loading and a grip strength 
test. Each physical test was separated by a 1-week rest for animal recovery. With $0 \%$ body-weight loading, the 3 groups differed in endurance $(p<0.05)$ (Fig. 2a). We progressively increased the body weight loading to $5 \%$ and $7.5 \%$. With $5 \%$ body weight loading, the endurance of the HEC group was greater than the LEC group by 4.48 -fold $(78.73 \pm 20.76$ vs $14.35 \pm 1.71$ min; $p=0.006$ ) (Fig. 2b). With $7.5 \%$ body-weight loading, the endurance of the LEC and MEC groups was lower than the HEC group by $52 \%$ and $36 \%$, respectively $(3.72 \pm 0.25$ and $4.95 \pm 0.41$ vs $7.75 \pm 0.92$ min) $(p<0.001$ and $p=0.005)$ (Fig. $2 c)$. In addition, the HEC group showed greater grip strength than the LEC group, by $14.7 \%(p<0.001)$ (Fig. $2 \mathrm{~d})$.

Table 1. Definition of groups and number of mice.

\begin{tabular}{lll}
\hline Groups & Range of swimming time & Amounts \\
\hline LEC & $<3$ hours & 24 \\
MEC & $3-5$ hours & 27 \\
HEC & $>5$ hours & 24 \\
\hline
\end{tabular}

LEC, low exercise capacity; MEC, medium exercise capacity; HEC, high exercise capacity

Table 2. General characteristics of growth and diet during experiments.

\begin{tabular}{llllllll}
\hline & $\begin{array}{l}\text { Baseline } \\
\text { BW }(\mathrm{g})\end{array}$ & $\begin{array}{l}\text { Week 1 } \\
\text { BW }(\mathrm{g})\end{array}$ & $\begin{array}{l}\text { Week 2 } \\
\text { BW }(\mathrm{g})\end{array}$ & $\begin{array}{l}\text { Week 3 } \\
\text { BW }(\mathrm{g})\end{array}$ & $\begin{array}{l}\text { Week 4 } \\
\text { BW }(\mathrm{g})\end{array}$ & $\begin{array}{l}\text { Diet } \\
(\mathrm{g})\end{array}$ & $\begin{array}{l}\text { Water } \\
(\mathrm{mL})\end{array}$ \\
\hline LEC & $28.6 \pm 0.5$ & $30.5 \pm 0.5$ & $33.1 \pm 0.5$ & $35.0 \pm 0.5$ & $36.5 \pm 0.5$ & $7.3 \pm 0.2$ & $7.7 \pm 0.4$ \\
MEC & $28.7 \pm 0.3$ & $30.7 \pm 0.3$ & $33.6 \pm 0.3$ & $35.7 \pm 0.4$ & $36.5 \pm 0.4$ & $7.7 \pm 0.4$ & $8.5 \pm 0.5$ \\
HEC & $28.5 \pm 0.4$ & $30.8 \pm 0.4$ & $33.9 \pm 0.4$ & $36.1 \pm 0.4$ & $37.1 \pm 0.5$ & $6.9 \pm 0.4$ & $7.8 \pm 1.2$ \\
\hline
\end{tabular}
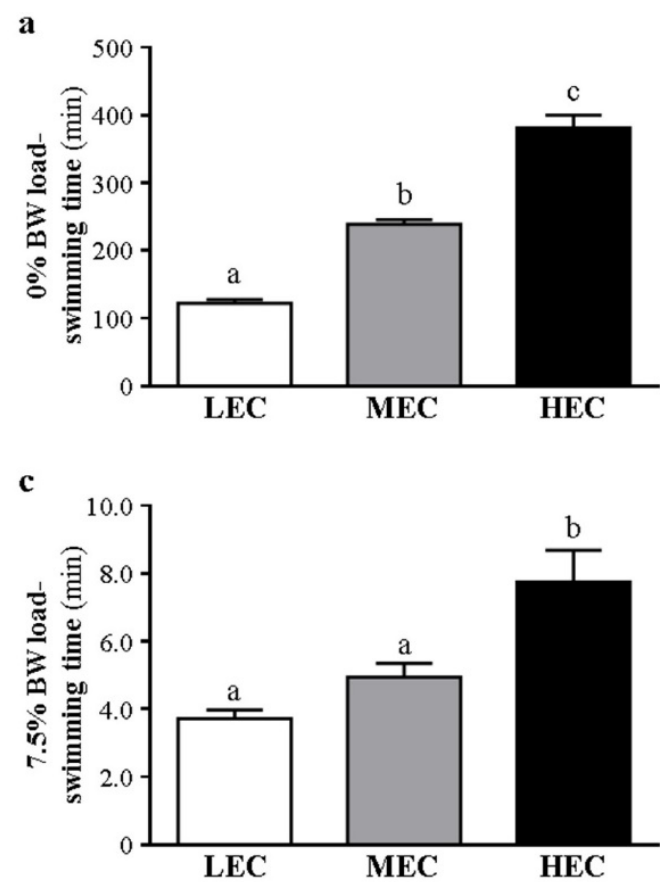

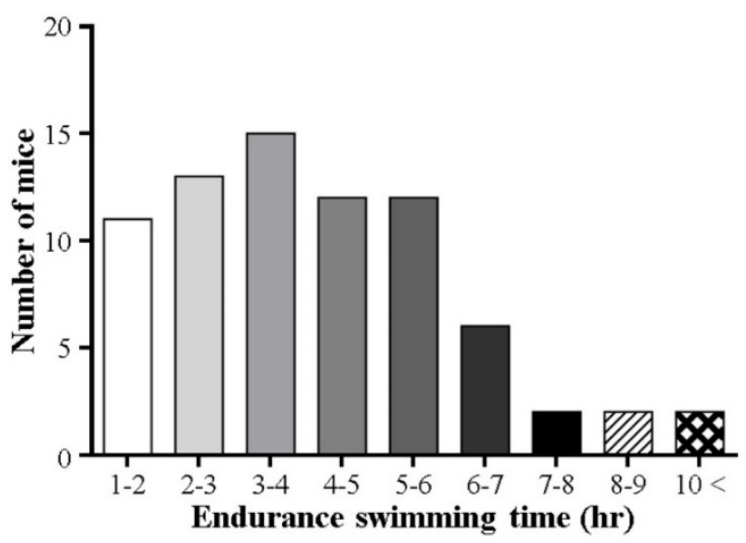

b

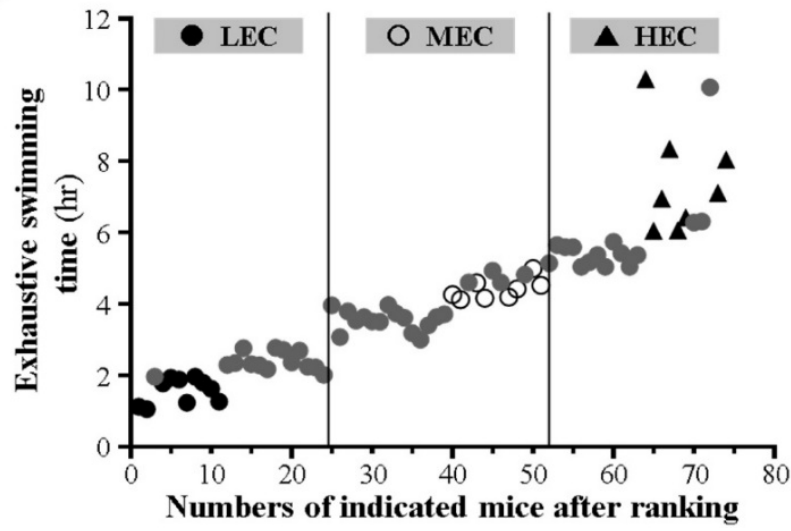

Figure. 1 Swimming exercise capacity in mice. a Capacity shown as endurance time by number of mice. b After capacity ranking with $0 \%$ loading, exercise capacity by defined LEC, MEC, and HEC mice. LEC, low exercise capacity $(<3$ hr); MEC, medium exercise capacity (3-5 hr); HEC, high exercise capacity ( $>5$ $\mathrm{hr})$.

b

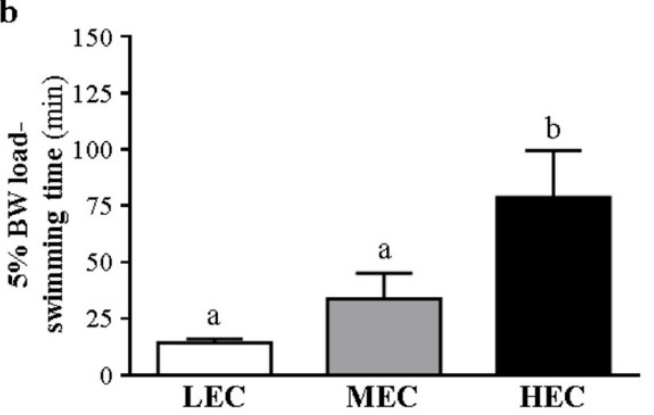

d

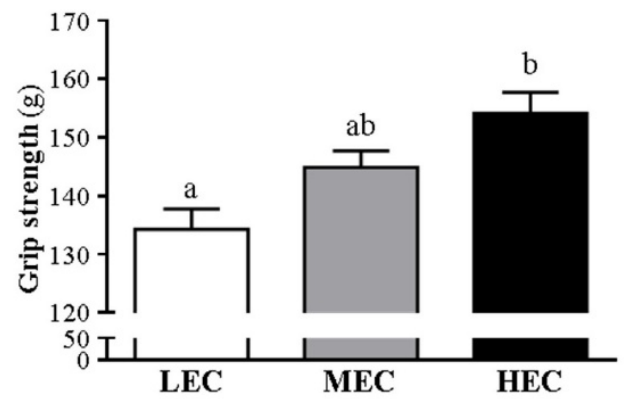

Figure. 2 Swimming time with different loadings and grip strength. The 3 capacity groups were challenged with different weight loading from $0 \%$ (a), $5 \%$ (b) and $7.5 \%$ (c). d Grip strength. Data are mean \pm SD from experiments. Bars with different letters (a, b, c) are significantly different at $p<0.05$. 
$\mathbf{a}$

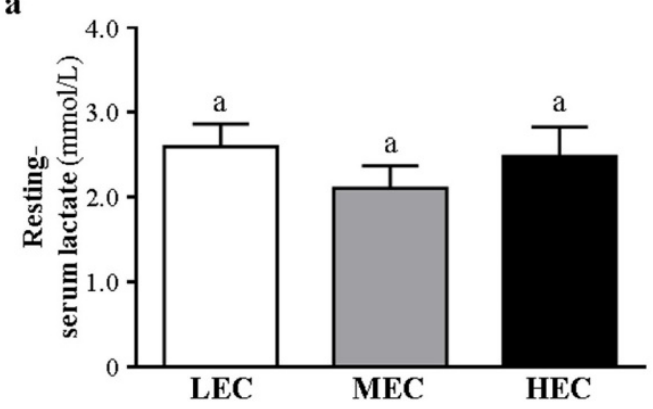

c

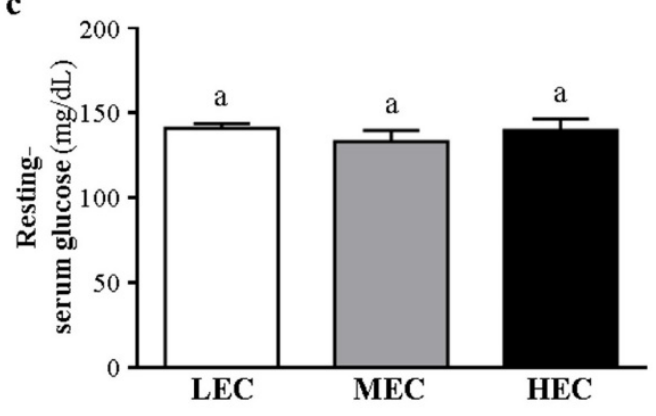

b

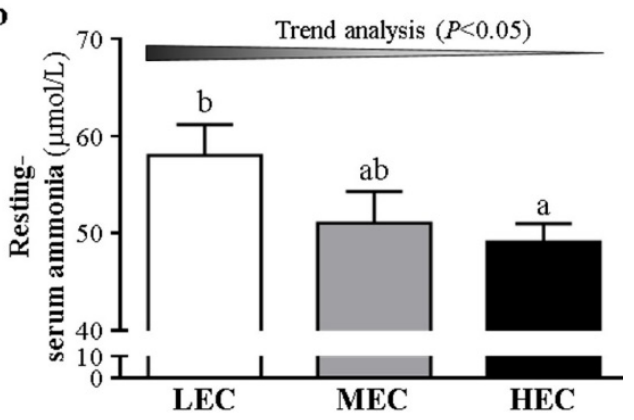

d

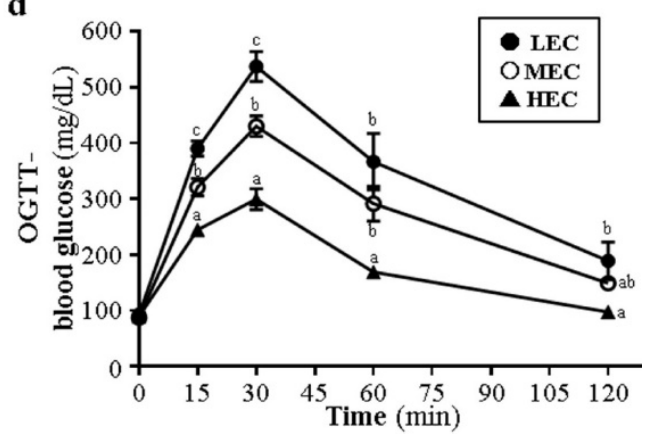

Figure. 3 Effect of different exercise capacity on serum levels of lactate (a); ammonia (b); glucose (c) during resting conditions. d The glucose tolerance was evaluated by oral glucose tolerance test (OGTT). Data are mean \pm SD from experiments. Bars or data points with different letters (a, b, c) are significantly different at $p<0.05$.

\section{Biochemical variables}

We examined fatigue-related biochemical indicators including lactate, ammonia, and glucose in the 3 groups to evaluate the fatigue status after an exhaustive exercise measured with the effects of functional nutrient supplements in previous studies $[7,9]$. During resting conditions, lactate level did not differ between the groups ( $p>0.05$, Fig. 3a). Ammonia level was lower, by $15.3 \%$, for the HEC than LEC group ( $p=0.0378$, Fig. $3 b$ ). Glucose level did not differ among the groups ( $p>0.05$, Fig. 3c). After a 15-min swimming challenge without loading (Fig. 4), lactate level was higher for the LEC group than MEC and HEC groups ( $p$ trend $<0.05)$. Ammonia level was lower, by $15.6 \%$, for the HEC than LEC group ( $p$ trend $<0.05$ ). Creatine kinase level was lower, by $30.5 \%$, for the HEC than LEC group ( $p$ trend <0.05). Lactate, ammonia, and creatine kinase levels were significantly decreased after physical challenge depending on capacity $(p<0.05)$.

\section{OGTT for glucose level}

After the administration of glucose $(2.5 \mathrm{~g} / \mathrm{kg})$ by oral gavage, blood was collected for glucose measurement (Fig. 3d). During resting, glucose level did not differ among the groups $(p>0.05)$. At 15 and 30 min, the level was higher for the LEC group than MEC and HEC groups $(p<0.05)$. At $60 \mathrm{~min}$, the level was higher for the LEC and MEC groups than HEC group ( $p<0.05)$. At $120 \mathrm{~min}$, the level was still higher for the LEC than HEC group $(p<0.05)$.

\section{Tissue weight and morphological examination}

Eight mice were sacrificed for morphological examination of weight of physical activity-related tissues at the end of the experiment. The groups did not differ in appearance of physical-activity related tissues ( $p>0.05$; Table 3 ) and the liver, lung and muscle did not differ among the groups (Fig. 4). However, the alveolus space was slightly greater for the HEC group than the other groups (Fig. 4).

Table 3. Organ weight (g) in the 3 mouse groups.

\begin{tabular}{lllllll}
\hline & Heart $(\mathrm{g})$ & Lung $(\mathrm{g})$ & Liver $(\mathrm{g})$ & Muscle $(\mathrm{g})$ & EAT $(\mathrm{g})$ & BAT $(\mathrm{g})$ \\
\hline LEC & $0.26 \pm 0.05$ & $0.22 \pm 0.06$ & $1.66 \pm 0.24$ & $0.18 \pm 0.02$ & $0.70 \pm 0.28$ & $0.17 \pm 0.04$ \\
MEC & $0.28 \pm 0.07$ & $0.23 \pm 0.04$ & $1.70 \pm 0.21$ & $0.20 \pm 0.02$ & $0.67 \pm 0.13$ & $0.19 \pm 0.04$ \\
HEC & $0.24 \pm 0.04$ & $0.24 \pm 0.06$ & $1.68 \pm 0.34$ & $0.18 \pm 0.02$ & $0.71 \pm 0.28$ & $0.18 \pm 0.03$ \\
\hline EAT, epididymal adipose tissue; BAT, brown adipose tissue.
\end{tabular}

\section{Correlation between physical activities and biochemical variables}

In terms of swimming endurance with different loading $(0 \%, 5 \%$, and $7.5 \%), 0 \%$ weight-loading endurance was moderately correlated with $5 \%$ and $7.5 \%$ weight-loading endurance $(\mathrm{r}=0.615, p=0.001$; $\mathrm{r}=0.542, p=0.006$; Fig. $5 \mathrm{a}$ and $5 \mathrm{~b})$ and $5 \%$ and $7.5 \%$ endurance were moderately correlated $(\mathrm{r}=0.606$, $p=0.002$, Fig. 5c). Furthermore, grip strength was 
moderately correlated with $0 \%$ weight-loading endurance $(\mathrm{r}=0.443, p=0.006$, Fig. $5 \mathrm{~d})$ but not $5 \%$ and $7.5 \%$ endurance $(\mathrm{r}=0.163, p=0.166 ; \mathrm{r}=0.099, p=0.409$; data not shown). Ammonia level was moderately negatively correlated with $7.5 \%$ loading $(\mathrm{r}=-0.447$, $p=0.029$; Fig. $6 a)$ and negatively but not significantly with $5 \%, 7.5 \%$, and grip strength $(\mathrm{r}=-0.391,-0.156$, and $-0.198 ; p>0.05$, data not shown). Lactate and ammonia level showed a moderate positive correlation at resting status ( $\mathrm{r}=0.598, p=0.002$; Fig. $6 \mathrm{~b}$ ).
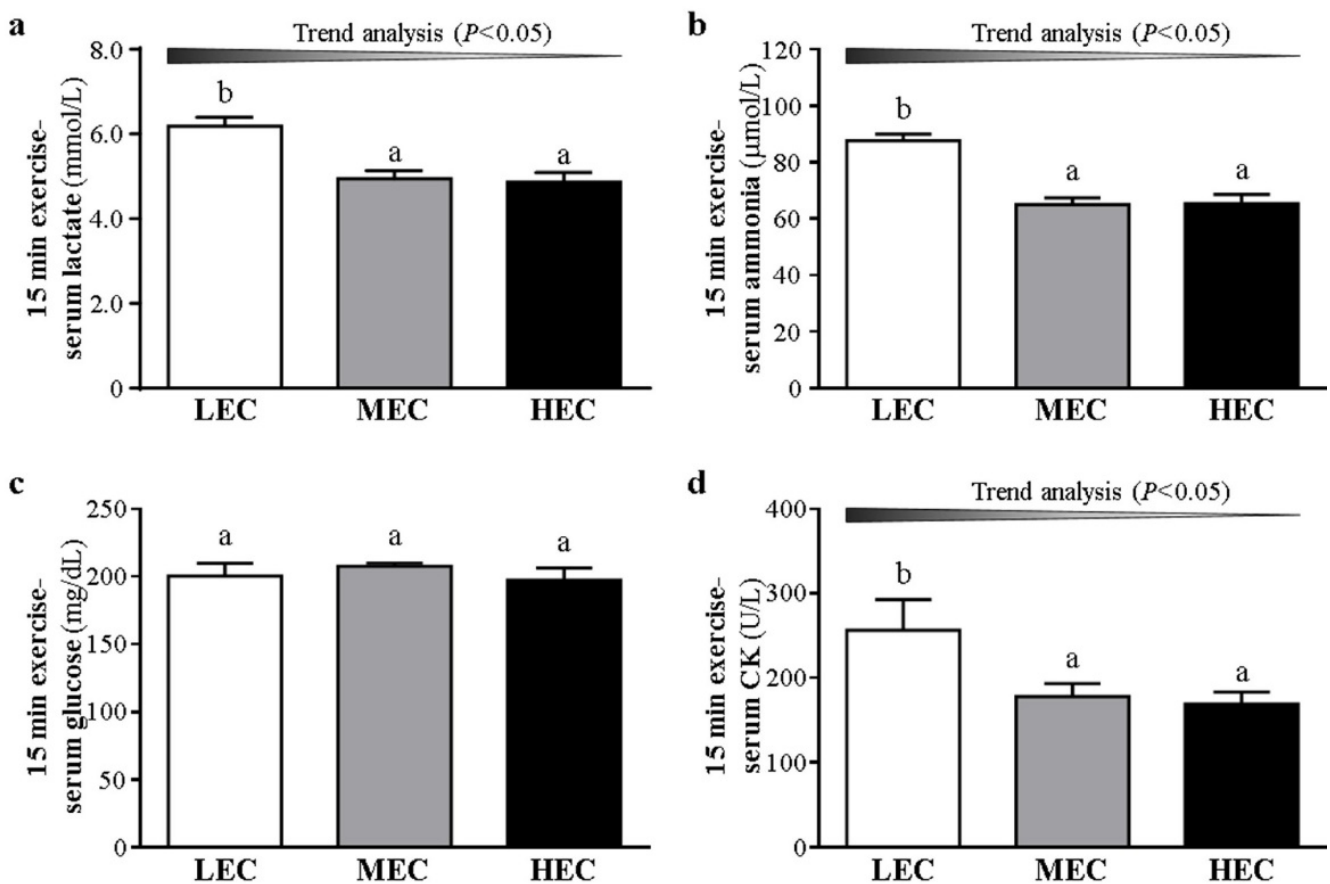

Figure. 4 Effect of the different exercise capacity on serum levels of lactate (a); ammonia (b); glucose (c) and CK (d) after a 15-min swimming exercise challenge without loading. The glucose tolerance was evaluated by OGTT (d). Data are mean \pm SD from experiments. Bars with different letters (a, b) are significantly different at $p<0.05$.

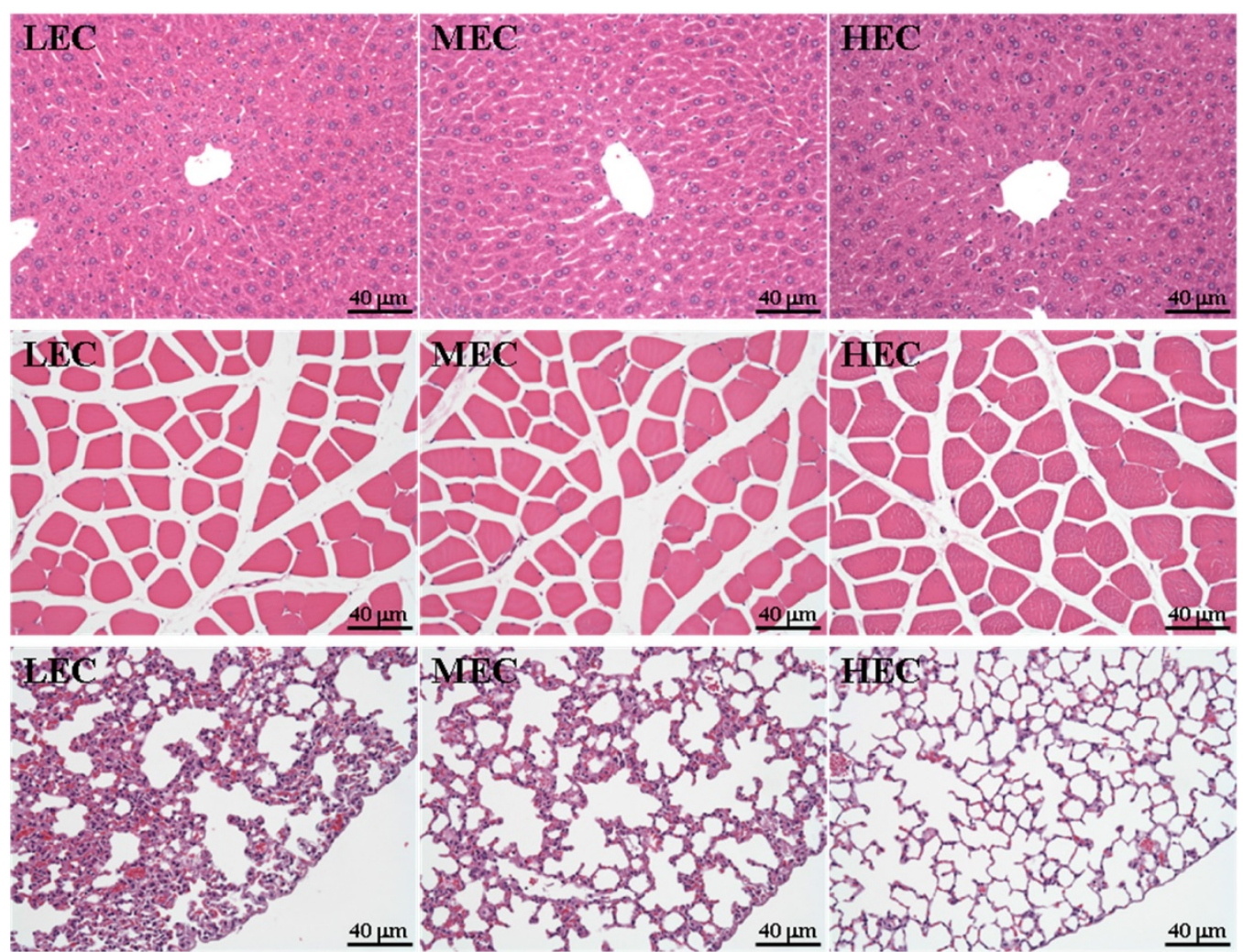

Figure. 5 Sections of the liver (top), muscle (middle) and lung (bottom) from LEC, MEC and HEC groups (H\&E stain). 
a

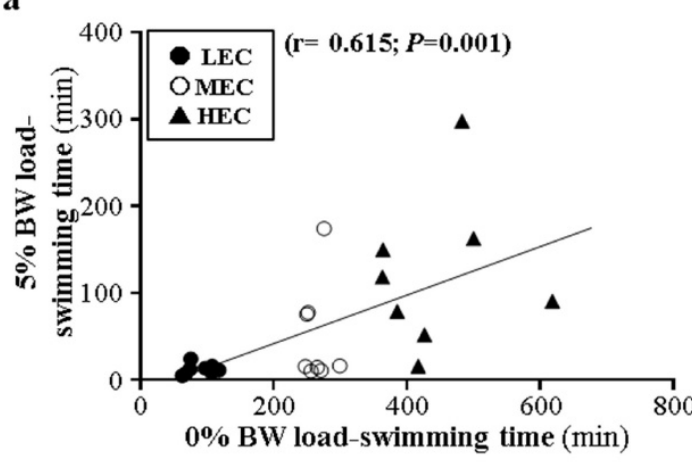

c

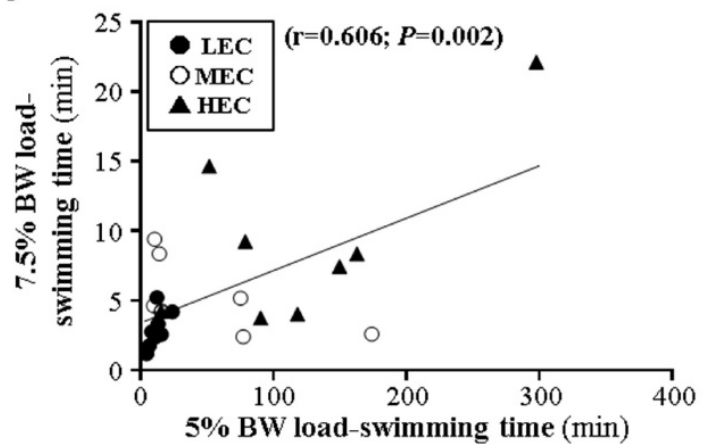

b

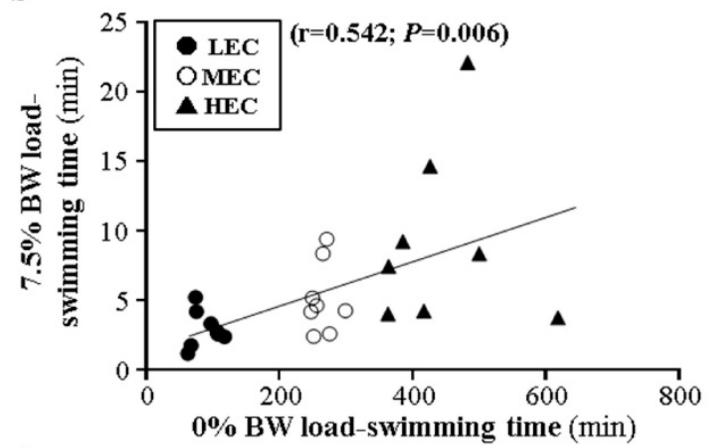

d

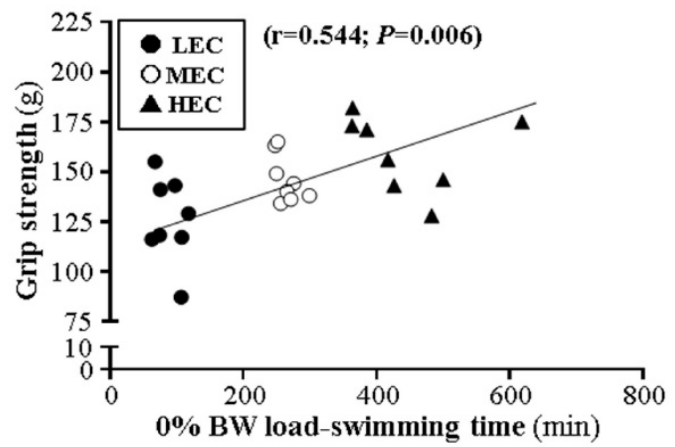

Figure. 6 Correlation of different physical activities for mice with different exercise capacity.

\section{Comment}

We previously found that the factors affecting mouse swimming endurance include weight loading, water temperature, and strain $[7,9]$. In this case, the factors were consistent with strain and water temperature, and congenital exercise performance showed a significant difference within the same strain according to different equivalent body-weight loading. The physiological or genetic factors may play important roles to cause this consistent difference.

The relationship between endurance capacity and voluntary activity level has been studied with mixed results. The variation in activity levels may rely on motive differences for being active as well as physical ability to increase for certain durations or particular intensities within and among species. In previous studies, 10 inbred strains were used to investigate the relationship between voluntary activities and treadmill endurance and showed that the 2 distribution patterns are not concordant and may be caused by different genetic contributions to these two phenotypes [23]. By using artificial selection/breeding for intrinsic aerobic endurance of genetically heterogeneous N:NIH rats, the high-capacity line showed 1.7-fold higher exhaustive time than the low-capacity line at generation 6 [17]. Hence, exercise capacity and voluntary activities could be affected by heritable factors [24].
Intrinsic endurance capacities have been evaluated by a treadmill running exercise in rats [17], and we used another aerobic exercise model, swimming, to distinguish intrinsic capacities. The capacities of the 3 mouse groups showed similar normal distribution (Fig. 1a) and ranking individuals by swimming time (Fig. 2b) showed results similar to that reported by Koch et al. [17]. The aerobic swimming model could also be another way to evaluate intrinsic endurance. As we showed, the outbred ICR strain mice showed intrinsic differences in exercise capacity in the founder population without artificial breeding (Fig. 1). After designating 3 groups as LEC, MEC, and HEC according to exhaustive swimming capacity with $0 \%$ weight loading (Table 1 ), these groups were challenged by $5 \%$ and $7.5 \%$ weight-loading tests, defined as moderate- and high-intensity exercise. The HEC group showed greater exercise endurance than the LEC group with $5 \%$ and $7.5 \%$ loading (Fig. 2). Therefore, intrinsic exercise endurance may not be affected by different exercise intensities. As well, grip strength was greater for the HEC than LEC group (Fig. 2d). Muscle strength can be improved by resistance training via muscular and neuromuscular adaption [25]. Therefore, the HEC mice may have better neuromuscular adaption or coordination to recruit more motor units for greater force production than the LEC group. However, further studies could 
investigate physiological differences. In the current study, we chose the ICR strain because this strain is widely used to evaluate nutrient supplements or interventions against fatigue or for exercise physiology [26-30]. Therefore, the individual capacity of experimental animals could influence the real effects caused by different interventions.

HEC mice could have greater glucose tolerance and maintain better homeostasis than the MEC and LEC groups (Fig. 3d). They could have better mechanisms to regulate glucose utilization for energy needs. The results were consistent with previous studies showing an association of high running capacity in rats and greater glucose uptake and oxidation as compared to low running capacity [31]. Bioinformatics analysis of skeletal muscle gene expression revealed that many of the genes upregulated in high-capacity rats were related to oxidative energy metabolism [32]. Biochemical variables including lactate, ammonia, and creatine kinase have been used to evaluate the physiological status of fatigue [7, 9, 33, 34]. We found resting ammonia level significantly different among the 3 mouse groups (Fig. 3b); ammonia content was lower in the HEC than LEC group. After the same 15-min swimming challenge, lactate, ammonia, and creatine kinase levels were lower in the HEC than LEC group, showing significant capacity-dependent effects (Fig. 4). In a previous study, rats with low capacity promptly became fatigued and had slow metabolic recovery after stimulated maximal muscle contractions [35]. Our current findings could also illuminate physiological metabolite production that could be affected by intrinsic capacities.

In previous study, artificial selection of rats for high or low exercise endurance showed that the low-capacity line was heavier than the high-capacity line by $16 \%$ to $20 \%$ [17]. We found no difference in body weight between mice with different exercise capacities (Table 2), and the proportion of adipose tissue, including EAT and BAT, did not differ (Table 3 ). The difference in weight and fat tissue proportion between previous studies and the current study may have been due to artificial breeding over several generations to establish the high- and low-capacity rats in the previous study. In the previous study, the low-capacity rats showed dysregulated muscle lipid metabolism, such as greater lipid accretion, reduced triglycerides lipase activity, greater level of non-esterified fatty acid, and triglyceride concentrations, as compared to high-capacity rats [36]. Our animals were a founder population, not artificially bred. Therefore, the genes related to lipid metabolism dysregulation were not evaluated in our case, but the weight and composition did not differ from previous results.

We found that only lung tissue showed a slight difference among groups, with greater alveolus space in the HEC group than in the LEC and MEC groups. Hemoperfusion and diffusion capacity may be elevated for oxygen being carried by red blood cells. In related reports, the pulmonary oxygen uptake was differentially affected by age and by exercise performance [37, 38]. In those morphological observations, the pulmonary development could be caused by the genetic variations of these 3 groups and could also provide different viewpoints to understand the developmental process under capacity evolution in future study.

The correlations among physiological biochemistry, physical activities, and capacity were little studied in previous studies. We found greater correlation with the HEC group than other groups and the capacity of the HEC group would not be affected by different intensities (Fig. 6a-c). The ammonia concentration was negatively correlated with physical activity with $7.5 \%$ weight loading and ammonia and lactate levels were positively correlated (Fig. 7). In a previous correlation study, grip strength could be related to the bone mineral density of the lumbar spine, femoral neck, and total body [39] and was reported as a predictor of nutrition status [40]. Aerobic capacity and grip strength are moderated by a different energy metabolism system. However, we found that grip strength was significantly correlated with intrinsic aerobic capacity (Fig. 6d). For future studies, individual exercise capacity could be evaluated by grip strength or ammonia concentration (Fig. 7a) before experimental intervention.

Here, we aimed to investigate the potential mediators and relationship that affects congenital exercise performance in an animal model with physical activity challenge from physiological and biochemical perspectives. Mice performed a non-loading and exhaustive swimming test and then were assigned to 3 groups by low, medium and high exercise capacity (LEC, MEC, HEC). Physical activity, including exhaustive swimming time and grip strength, was greater for HEC than other mice. Swimming performance and grip strength were moderately correlated between groups. Resting serum ammonium level was moderately correlated with performance with a $7.5 \%$ weight load and with lactate level $(p<0.05)$. The pulmonary morphology of the HEC group seemed to indicate benefits for aerobic exercise. Mice showed congenital exercise performance, which was significantly correlated with different physical challenges and biochemical variables. This study may have implications for interference in intrinsic characteristics. 
a

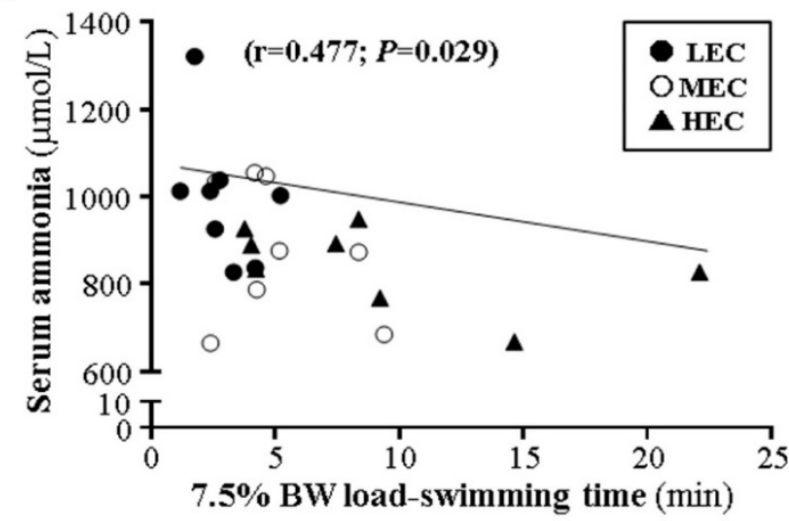

b

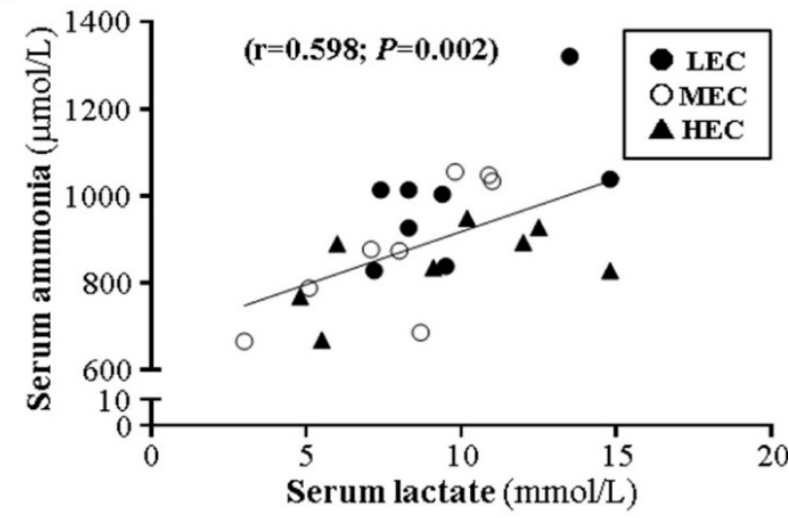

Figure. 7 Correlation of ammonia level and $7.5 \%$ weight loading (a) and lactate level (b) for mice with difference exercise capacity.

\section{Conclusions}

We demonstrated an intrinsic difference in exercise performance in outbred mice, which have been widely used in sport science for physiological research. The intrinsic differences might cause experimental variations or improper interpretations of the effects of interventions or supplements. We provide correlation findings for physical activities and basic biochemical variables for related references. In practical application, researchers could investigate the effect of interventions on experimental animals without influences caused by intrinsic differences in physical activities or physiology. However, these intrinsic differences in exercise performance also leave many questions to answer. In the future, these populations with different exercise capacities could be studied in terms of genomics and proteomics to provide a better understanding of the physiological differences in exercise performance.

\section{Acknowledgements}

The corresponding author Huang Chi-Chang acknowledges the Ministry of Science and Technology (MOST) of Taiwan, the successor to the National
Science

Council

(grant

no. MOST-104-2628-H-179-001-MY3), for financial support. The authors thank Laura Smales (BioMedEditing, Toronto, Canada) for her careful editing of the manuscript, Miss Jou-Chi Tang for providing technical assistance in animal experiments, and Dr. Chin-Chao Chiu, a pathological veterinarian, for interpreting the pathological data.

\section{Authors' contributions}

Wen-Ching Huang, Ying-Ju Chen and Chi-Chang Huang designed the experiments. Wen-Ching Huang, Yi-Ju Hsu, and Li Wei carried out the laboratory experiments. Wen-Ching Huang, Li Wei, and Chi-Chang Huang analyzed the data, interpreted the results, prepared figures, and wrote the manuscript. Chi-Chang Huang and Ying-Ju Chen contributed reagents, materials and analysis platforms. All authors read and approved the final manuscript.

\section{Competing Interests}

The authors have declared that no competing interest exists.

\section{References}

1. Welsh CE, Miller DR, Manly KF, Wang J, McMillan L, Morahan G, Mott R, Iraqi FA, Threadgill DW, de Villena FP.. Status and access to the Collaborative Cross population. Mamm Genome. 2012;23:706-12.

2. Yalcin B, Flint J. Association studies in outbred mice in a new era of full-genome sequencing. Mamm Genome. 2012;23:719-726.

3. Kang CK, Hah DS, Kim CH, Kim E, Kim JS. Evaluation of the genotoxicity of extracts of Houttuynia cordata Thunb. Am J Chin Med. 2012;40:1019-32.

4. Nguyen TM, Nakamura H, Wakabayashi A, Kanagawa T, Koyama S, Tsutsui $\mathrm{T}$, Hamasaki T, Kimura T. Estimation of mouse fetal weight by ultrasonography: application from clinic to laboratory. Lab Anim. 2012;46:225-30.

5. Guo X, Huang X, Wu YS, Liu DH, Lu HL, Kim YC, Xu WX. Down-regulation of hydrogen sulfide biosynthesis accompanies murine interstitial cells of Cajal dysfunction in partial ileal obstruction. PLoS One. 2012;7:e48249.

6. Huang CC, Huang WC, Yang SC, Chan CC, Lin WT. Ganoderma tsugae Hepatoprotection against Exhaustive exercise-induced liver injury in rats. Molecules. 2013;18:1741-54.

7. Huang CC, Hsu MC, Huang WC, Yang HR, Hou CC. Triterpenoid-Rich Extract from Antrodia camphorata Improves Physical Fatigue and Exercise Performance in Mice. Evid Based Complement Alternat Med. 2012;2012:364741.

8. Xu C, Lv J, Lo YM, Cui SW, Hu X, Fan M. Effects of oat $\beta$-glucan on endurance exercise and its anti-fatigue properties in trained rats. Carbohydr Polym. 2013;92:1159-65.

9. Wang SY, Huang WC, Liu CC, Wang MF, Ho CS, Huang WP, Hou CC, Chuang HL, Huang CC. Pumpkin (Cucurbita moschata) fruit extract improves physical fatigue and exercise performance in mice. Molecules. 2012;17:11864-76.

10. Ikarashi N, Fukazawa Y, Toda T, Ishii M, Ochiai W, Usukura M, Sugiyama K. Effect of Conclevan on endurance capacity in mice. Biol Pharm Bull. 2012;35:231-238.

11. Takeda K, Machida M, Kohara A, Omi N, Takemasa T. Effects of citrulline supplementation on fatigue and exercise performance in mice. J Nutr Sci Vitaminol. 2011;57:246-50.

12. Huang LZ, Huang BK, Ye Q, Qin LP. Bioactivity-guided fractionation for anti-fatigue property of Acanthopanax senticosus. J Ethnopharmaco. 2011;133:213-19.

13. Sahlin K. Metabolic factors in fatigue. Sports Med. 1992;13:99-107.

14. Cairns SP. Lactic acid and exercise performance: culprit or friend? Sports Med. 2006;36:279-291.

15. Banister EW, Cameron BJ. Exercise-induced hyperammonemia: peripheral and central effects. Int J Sports Med. 1990;11:S129-142.

16. Falavigna G, Alves de Araújo JJr, Rogero MM, Pires IS, Pedrosa RG, Martins EJr, Alves de Castro I, Tirapegui J. Effects of diets supplemented with 
branched-chain amino acids on the performance and fatigue mechanisms of rats submitted to prolonged physical exercise. Nutrients. 2012;4:1767-80.

17. Koch LG, Britton SL. Artificial selection for intrinsic aerobic endurance running capacity in rats. Physiol Genomics. 2001;5:45-52.

18. Waters RP, Pringle RB, Forster GL, Renner KJ, Malisch JL, Garland TJr, Swallow JG. Selection for increased voluntary wheel-running affects behavior and brain monoamines in mice. Brain Research. 2013;1508:9-22.

19. DeMarco VG, Johnson MS, Ma L, Pulakat L, Mugerfeld I, Hayden MR, Garro M, Knight W, Britton SL, Koch LG, Sowers JR. Overweight female rats selectively breed for low aerobic capacity exhibit increased myocardial fibrosis and diastolic dysfunction. Am J Physiol Heart Circ Physiol. 2012;302:1667-1682.

20. Koch LG, Kemi OJ, Qi N, Leng SX, Bijma P, Gilligan LJ, Wilkinson JE, Wisløff H, Høydal MA, Rolim N, Abadir PM, van Grevenhof EM, Smith GL, Burant $\mathrm{CF}$, Ellingsen $\mathrm{O}$, Britton SL, Wisløff U. Intrinsic aerobic capacity sets a divide for aging and longevity. Circ Res. 2011;109:1162-1172.

21. Beighley PE, Zamir M, Wentz RJ, Koch LG, Britton SL, Ritman EL. Vascularity of myocardium and gastrocnemius muscle in rats selectively bred for endurance running capacity. Physiol Genomics. 2013;45:119-125.

22. Ho TJ, Huang CC, Huang CY, Lin WT. Fasudil, a Rho-kinase inhibitor, protects against excessive endurance exercise training-induced cardiac hypertrophy, apoptosis and fibrosis in rats. Eur J Appl Physiol. 2012;112:2943-2955.

23. Lightfoot JT, Turner MJ, Daves M, Vordermark A, Kleeberger SR. Genetic influence on daily wheel running activity level. Physiol Genomics. 2004;19:270-276.

24. Meek TH, Lonquich BP, Hannon RM, Garland TJr. Endurance capacity of mice selectively bred for high voluntary wheel running. J Exp Biol. 2009;212:2908-2917.

25. Folland JP, Williams AG. The adaptations to strength training: morphological and neurological contributions to increased strength. Sports Med. 2007;37:145-168

26. Sheng R, Xu X, Tang Q, Bian D, Li Y, Qian C, He X, Gao X, Pan R, Wang C, Luo Y, Xia Y, Dai Y. Polysaccharide of radix pseudostellariae improves chronic fatigue syndrome induced by poly I:C in mice. Evid Based Complement Alternat Med. 2011;2011:840516.

27. Jung HY, Lee AN, Song TJ, An HS, Kim YH, Kim KD, Kim IB, Kim KS, Han BS, Kim CH, Kim KS, Kim JB. Korean mistletoe (Viscum album coloratum) extract improves endurance capacity in mice by stimulating mitochondrial activity. J Med Food. 2012;15:621-628

28. Kim NH, Moon PD, Pak SC, Kim HM, Jeong HJ. Anti-fatigue effect of Zizania caudiflora (Turczaninow) Nakai. Am J Chin Med. 2012;40:111-120.

29. Carter LG, Lewis KN, Wilkerson DC, Tobia CM, Ngo Tenlep SY, Shridas P, Garcia-Cazarin ML, Wolff G, Andrade FH, Charnigo RJ, Esser KA, Egan JM, de Cabo R, Pearson KJ. Perinatal exercise improves glucose homeostasis in adult offspring. Am J Physiol Endocrinol Metab. 2012;303:1061-1068.

30. Huang WC, Lin CI, Chiu CC, Lin YT, Huang WK, Huang HY, Huang CC. Chicken essence improves exercise performance and ameliorates physical fatigue. Nutrients. 2014;6:2681-2696.

31. Rivas DA, Lessard SJ, Saito M, Friedhuber AM, Koch LG, Britton SL, Yaspelkis BB 3rd, Hawley JA. Low intrinsic running capacity is associated with reduced skeletal muscle substrate oxidation and lower mitochondrial content in white skeletal muscle. Am J Physiol Regul Integr Comp Physiol. 2011;300:835-843.

32. Kivelä R, Silvennoinen M, Lehti M, Rinnankoski-Tuikka R, Purhonen T, Ketola T, Pullinen K, Vuento M, Mutanen N, Sartor MA, Reunanen H, Koch LG, Britton SL, Kainulainen H. Gene expression centroids that link with low intrinsic aerobic exercise capacity and complex disease risk. FASEB J. 2010;24:4565-74.

33. Wu RE, Huang WC, Liao CC, Chang YK, Kan NW, Huang CC. Resveratrol protects against physical fatigue and improves exercise performance in mice. Molecules. 2013;18:4689-4702.

34. Chen WC, Huang WC, Chiu CC, Chang YK, Huang CC. Whey Protein Improves Exercise Performance and Biochemical Profiles in Trained Mice. Med Sci Sports Exerc. 2014;46:1517-1524.

35. Torvinen $S$, Silvennoinen $M$, Piitulainen $H$, Närväinen J, Tuunanen $P$, Gröhn O, Koch LG, Britton SL, Kainulainen H. Rats bred for low aerobic capacity become promptly fatigued and have slow metabolic recovery after stimulated, maximal muscle contractions. PLoS One. 2012;7:e48345.

36. Spargo FJ, McGee SL, Dzamko N, Watt MJ, Kemp BE, Britton SL, Koch LG, Hargreaves M, Hawley JA. Dysregulation of muscle lipid metabolism in rats selectively bred for low aerobic running capacity. Am J Physiol Endocrinol Metab. 2007;292:1631-1636.

37. Dempsey JA, McKenzie DC, Haverkamp HC, Eldridge MW. Update in the understanding of respiratory limitations to exercise performance in fit, active adults. Chest. 2008;134:613-22.

38. Stickland MK, Butcher SI, Marciniuk DD, Bhutani M. Assessing exercise limitation using cardiopulmonary exercise testing. Pulm Med. 2012;2012:824091.

39. Marin RV, Pedrosa MA, Moreira-Pfrimer LD, Matsudo SM, Lazaretti-Castro M. Association between lean mass and handgrip strength with bone mineral density in physically active postmenopausal women. J Clin Densitom. 2010;13:96-101.

40. Flood A, Chung A, Parker H, Kearns V, O'Sullivan TA. The use of hand grip strength as a predictor of nutrition status in hospital patients. Clin Nutr. 2014;33:106-114 